\title{
CARACTERIZAÇÃO ESPECTRO-TEMPORAL DE PASTAGENS \\ DO TRIÂNGULO MINEIRO UTILIZANDO \\ DADOS MODIS EVI2 (2000-2010)
}

\section{Spectral and Temporal Characterization of Pastures from Triangulo Mineiro, State of Minas Gerais, using data MODIS EVI2 (2000-2010)}

\author{
Viviane Silveira Anjos \\ Universidade de Brasília, Brasília/DF \\ vivisanjos@gmail.com \\ Edson Eyji Sano \\ Embrapa Cerrados, Brasília/DF. Bolsista CNPq \\ sano@cpac.embrapa.br \\ Heleno da Silva Bezerra \\ Embrapa Cerrados, Brasília/DF \\ heleno@cpac.embrapa.br \\ Roberto Rosa \\ rrosa@ufu.br
}

Professor Associado, Instituto de Geografia, UFU. Bolsista CNPq

Artigo recebido em 25/02/2012 e aceito para publicação em 21/02/2013

RESUMO: Pastagens cultivadas são as classes de cobertura de terras mais representativas de três municípios (Araguari, Uberaba e Uberlândia) que fazem parte da mesorregião do Triângulo Mineiro no extremo oeste de Minas Gerais. O objetivo dessa pesquisa foi conduzir estudos de caracterização espectro-temporal das pastagens cultivadas dos três municípios acima referidos por meio de séries temporais do produto MODIS EVI2 (índice de vegetação realçado do sensor Moderate Resolution Imaging Spectrorradiometer do período 2000-2010). Para isso, foram selecionadas 25 áreas de pastagens cultivadas da espécie Brachiaria e mais três áreas ocupadas por cultura agrícola, floresta estacional e savana gramíneo-lenhosa. Séries temporais MODIS EVI2 foram obtidas através do portal do Instituto Nacional de Pesquisas Espaciais (INPE) e analisadas por meio de gráfico de linhas, análise de agrupamento hierárquico e somatório dos índices de vegetação. Resultados indicaram que é possível discriminar pastagens cultivadas de outras classes de cobertura de terras da região e que a somatória de valores EVI2 de toda a série histórica pode aumentar consideravelmente essa capacidade de discriminação. A maioria das pastagens cultivadas apresentou assinaturas espectro-temporais distintas. A substituição da pastagem por outros tipos de cobertura de terras (por exemplo, cultura agrícola e vice-versa) é facilmente identificada na série temporal considerada. Palavras chave: Índice de vegetação, sensoriamento remoto, mapeamento de pastagens.

ABSTRACT: Cultivated pastures are the most representative land use and land cover classes in the three municipalities (Araguari, Uberaba and Uberlândia) belonging to the Triangulo Mineiro mesorregion in the western part of Minas Gerais State. The objective of this research was to conduct the spectral and temporal charac- 
terization of cultivated pastures from the above mentioned municipalities through MODIS EVI2 time series (enhanced vegetation index from Moderate Resolution Imaging Spectroradiometer, time series of 2000-2010). We selected 25 sampling areas of cultivated pastures (Brachiaria) and three additional areas occupied with cropland, forestland, and grassland. Temporal series of MODIS EVI2 were obtained from the National Space Research Institute (INPE) data gateway and analyzed via graphs of lines, cluster analysis and cumulative vegetation indices. Results showed that it is possible to discriminate cultivated pastures from other land use and land cover classes and that the cumulative EVI2 data involving entire temporal series can increase this discrimination capability substantially. Most of the cultivated pastures presented distinct spectro-temporal signatures. Changes of cultivated pastures for other land use classes (for instance, croplands and vice-versa) are easily identified in the considered temporal series.

Keywords: Vegetation index, remote sensing, pasture mapping.

\section{INTRODUÇÃO}

Pastagens cultivadas são a classe de cobertura de terras mais representativa na região formada pelos municípios de Araguari, Uberaba e Uberlândia da mesorregião do Triângulo Mineiro, no extremo oeste de Minas Gerais. De acordo com o mapeamento de cobertura vegetal natural e antrópica dos biomas brasileiros de 2002, coordenado pelo Ministério do Meio Ambiente no âmbito do Projeto de Conservação e Utilização Sustentável da Diversidade Biológica Brasileira (PROBIO) (SANO et al., 2010; MMA, 2012), as pastagens cultivadas ocupam mais de $50 \%$ do território formado por esses três municípios.

Estudos de discriminação de classes de cobertura de terras no Triângulo Mineiro têm utilizado imagens de satélite de uma única data (e.g., BRITO; PRUDENTE, 2005a, 2005b; MELO; FEHR, 2010). Entretanto, a discriminação, mapeamento e monitoramento de pastagens cultivadas dessa região com imagens de satélite de uma única data não é uma tarefa simples por causa da sua elevada dinâmica anual e sazonal na área de estudo. Ao longo do tempo, algumas dessas pastagens são substituídas por culturas agrícolas ou substituem a vegetação nativa. Elas apresentam ainda alta sensibilidade à forte sazonalidade climática da região (tipicamente, seis meses de chuva e seis meses de seca), podendo estar verdes ou secas, dependendo do mês do ano. Isso significa que, ao longo do ano, uma mesma área de pastagem cultivada pode apresentar padrões espectrais total- mente distintas. Como conseqüência, o mapeamento e monitoramento de pastagens cultivadas dessa região por meio de imagens de satélite de uma única data não é uma tarefa muito simples.

Uma alternativa para essas limitações é o uso de satélites com elevada resolução temporal. Esse é o caso, por exemplo, do sensor Moderate Resolution Imaging Spectroradiometer (MODIS), a bordo das plataformas Terra e Aqua (Justice et al., 2002), o qual foi lançado para fornecer observações globais de longa duração da superfície terrestre com uma periodicidade de 1-2 dias e resoluções espaciais moderadas (250 $\mathrm{m}-1 \mathrm{~km}$ ) (Miura et al., 2008). Além dessa elevada resolução temporal, outra vantagem dos dados do MODIS é a disponibilidade de produtos prontos para serem analisados, isto é, sem a necessidade de pré-processamentos adicionais pelo analista. Dois desses produtos são o índice de vegetação normalizado pela diferença (NDVI) e o índice de vegetação realçado (EVI), disponibilizados a cada 16 dias com uma resolução espacial de 250 metros (HUETE et al., 2002). $\mathrm{O}$ potencial das imagens multitemporais do MODIS para discriminar classes de cobertura de terras da bacia do rio Araguari-MG foi demonstrado por Rosendo e Rosa (2005). O objetivo desse estudo foi conduzir uma caracterização espectro-temporal das pastagens cultivadas representativas do Triângulo Mineiro por meio de dados históricos do MODIS EVI2 de 2000 a 2010. 


\section{MATERIAIS E MÉTODOS}

A área de estudo corresponde aos municípios de Araguari, Uberaba e Uberlândia cobertos pelo bioma Cerrado, localizada aproximadamente entre os paralelos $18^{\circ} 20^{\prime}$ e $20^{\circ} 10^{\prime}$ de latitude sul e entre os meridianos $47^{\circ} 35^{\prime}$ e $48^{\circ} 50^{\prime}$ de longitude oeste, na mesorregião do Triângulo Mineiro, extremo oeste de Minas Gerais (Figura 1).

Figura 1: Localização da área de estudo (municípios de Araguari, Uberlândia e Uberaba, de cima para baixo) no estado de Minas Gerais.

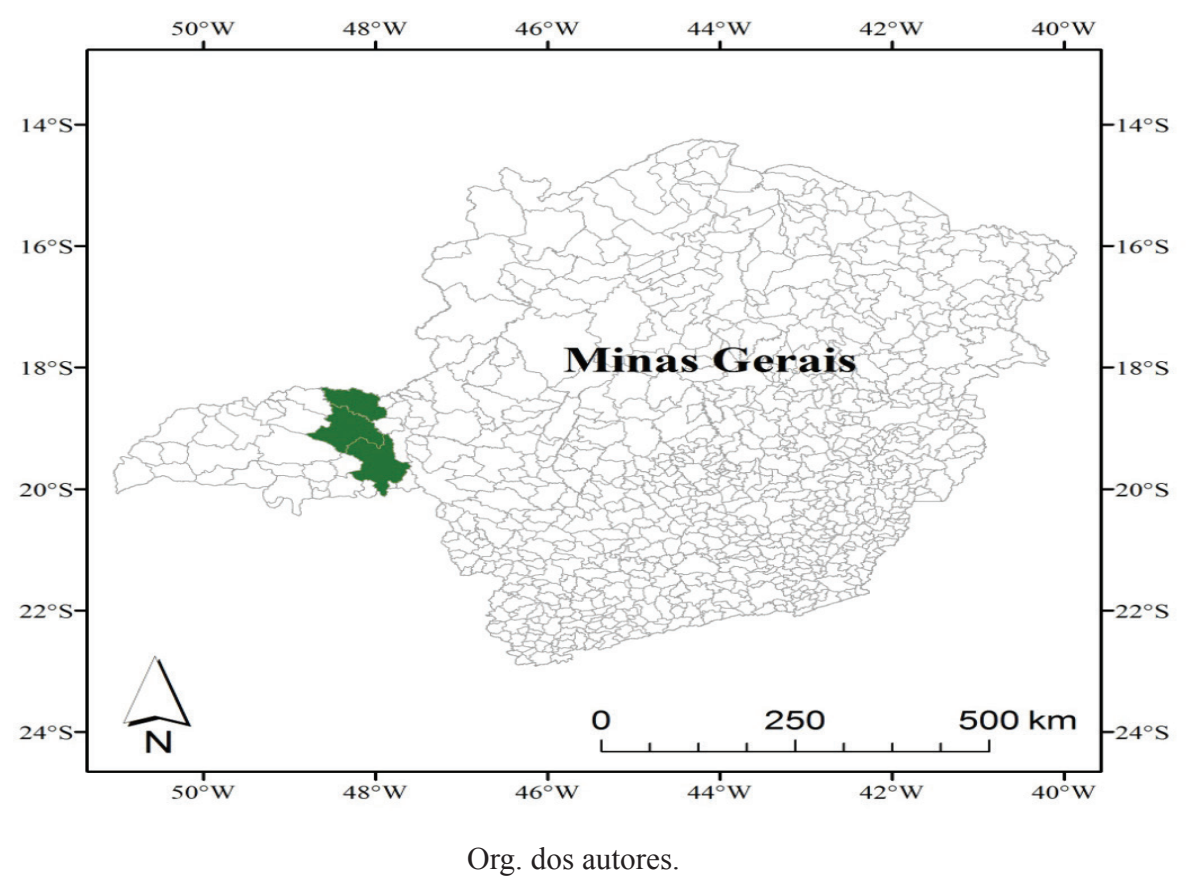

Os três municípios possuem uma área total de aproximadamente 1,14 milhões de hectares, enquanto a parte coberta pelo Cerrado ocupa cerca de um milhão de hectares. As duas classes de cobertura de terras mais representativas da área de estudo são as pastagens cultivadas e as culturas agrícolas, com 507.000 hectares e 190.000 hectares, respectivamente (dados derivados de arquivos em formato shape disponibilizados pelo MMA, 2012). O regime de precipitação típico da região pode ser demonstrado a partir dos dados diários de chuva da estação pluviométrica localizada no município de Uberaba (estação Uberabinha, $22^{\circ} 22^{\prime} \mathrm{S}$

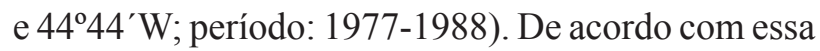
estação, a precipitação média anual da região é de aproximadamente $1.600 \mathrm{~mm}$, com período chuvoso concentrado nos meses de outubro a março e o período seco concentrado de abril a setembro.

As áreas de pastagens cultivadas mapeadas pelo projeto PROBIO com imagens do satélite Landsat de 2002 foram atualizadas para 2010. Para isso, quatro novas cenas do Landsat TM foram obtidas do portal do Instituto Nacional de Pesquisas Espaciais (INPE) (INPE, 2011; Tabela 1). As cenas foram georreferenciadas com base em imagens geocover (imagens ortorretificadas do Landsat ETM+ disponíveis no portal da Universidade de Maryland), no sistema de projeção UTM (zona $23 \mathrm{Sul}$ ) e referencial geodésico WGS 84, com erro quadrático médio inferior a 1 pixel. Essas imagens georreferenciadas foram, em seguida, mosaicadas e recortadas para a área de estudo. Os polígonos correspondentes a áreas de pastagens foram delimitadas visualmente na tela do monitor de computador por meio de um aplicativo de sistema de informações geográficas. A interpretação visual foi feita com base na composição colorida RGB das bandas 3 , 4 e 5 do referido sensor. 
Tabela 1. Órbita/ponto e datas de passagem das imagens do satélite Landsat TM utilizada para atualização do mapa de pastagens cultivadas da área de estudo.

\begin{tabular}{cc}
\hline Orbita/Ponto & Data da passagem \\
\hline $220 / 073$ & $27 / 05 / 2010$ \\
$220 / 074$ & $27 / 05 / 2010$ \\
$221 / 073$ & $02 / 05 / 2010$ \\
$221 / 074$ & $02 / 05 / 2010$ \\
\hline
\end{tabular}

Org. dos autores.

Com base nesse mapa de pastagens cultivadas da área de estudo, uma campanha de campo foi feita no período de 11 a 15 de abril de 2011 para selecionar áreas de pastagens representativas da área de estudo. Foram selecionados 25 pontos ao longo das principais rodovias da região. Para cada ponto, foram obtidas as coordenadas GPS e fotografias digitais panorâmicas, além da identificação da espécie de pastagem. Nesse conjunto de pontos, procuraram-se incluir aquelas pastagens mais extensas e com a maior variação possível em termos de produção de biomassa.

Para cada um dos 25 pontos selecionados, foram obtidos valores quinzenais de EVI2 da série histórica (2000-2010) do MODIS disponível no portal do INPE (http://www.dpi.inpe.br/laf). Nesse portal, é disponibilizada uma ferramenta para visualização instantânea das séries temporais derivadas de imagens de satélite dos sensores MODIS e do TRMM (Tropical Rainfall Measuring Mission) para dar suporte a estudos e análises de mudança de uso e cobertura da terra (FREITAS, et. al., 2011). Estas séries estão integradas às funcionalidades do globo virtual do Google Maps ${ }^{\mathrm{TM}}$.

Os dados do EVI2 disponibilizados nesse portal são derivados do produto MOD13 (interpolados), coleção 5, com resolução espacial de 250 metros e resolução temporal de 16 dias. O EVI2 é calculado pela seguinte fórmula (Jiang et al., 2008):

$$
E V I 2=2,5 \frac{\rho_{\text {NIR }}-\rho_{\text {RED }}}{\rho_{\text {NIR }}+2,4 \rho_{\text {RED }}+1}
$$

onde $P_{N I R}=$ reflectância na banda do infravermelho próximo; e $P_{R E D}=$ reflectância na banda do vermelho.

No EVI2, ao contrário do EVI proposto por Huete et al. (2002), não há inclusão da banda espectral na faixa do azul, cuja função é minimizar ruídos e incertezas associadas com a presença de aerossóis na atmosfera. De acordo com Jiang et al. (2008), EVI2 e EVI permanecem equivalentes do ponto de vista funcional, especialmente nos casos em que a qualidade dos dados do MODIS é boa. O número de pixels do MODIS EVI2 de cada pastagem variou em função da sua extensão. Procurou-se selecionar somente os pixels "puros", isto é, aqueles situados inteiramente dentro da área de pastagem. Em seguida, calculou-se a média aritmética simples para gerar uma única série temporal de cada pastagem.

A série temporal de um ponto de pastagem cultivada (Ap), selecionada aleatoriamente da lista de 25 pontos, foi comparada ainda com as correspondentes séries de três classes de cobertura de terras mais representativas da área de estudo: culturas agrícolas (Ac), floresta estacional semidecidual $(\mathrm{Fa})$ e savana gramíneo-lenhosa ( $\mathrm{Sg}$ ). No sistema de classificação de vegetação do Cerrado proposto por Ribeiro e Walter (2008), Fa corresponde à mata de galeria/cerradão e $\mathrm{Sg}$ ao campo limpo. Os três pontos adicionais foram selecionados com base nos arquivos em formato shape disponibilizados pelo MMA (2012) e nas imagens de alta resolução espacial disponíveis no programa Google Earth ${ }^{\mathrm{TM}}$. As coordenadas geográficas dos quatro pontos encontram-se na Tabela 2. As séries temporais foram obtidas por um único pixel de 250 metros, representativo de cada classe considerada.

Tratamentos estatísticos de séries temporais envolveram a construção de gráfico de linhas, somatória de valores individuais de EVI2 de cada data e análise de agrupamento hierárquico (cluster analysis; MOITA NETO; MOITA, 1998). 
Tabela 2. Latitude e longitude das séries temporais do MODIS EVI2 correspondentes as seguintes classes de cobertura de terras que foram comparadas entre si: pastagem cultivada, cultura agrícola, floresta estacional semidecidual e savana gramíneo-lenhosa.

\begin{tabular}{ccc}
\hline Classe & Latitude & Longitude \\
\hline Pastagem cultivada & $-19,7489$ & $-48,2325$ \\
Cultura agrícola & $-19,2241$ & $-48,1144$ \\
Floresta estacional semidecidual & $-19,0377$ & $-48,5768$ \\
Savana gramíneo-lenhosa & $-19,2914$ & $-48,0111$ \\
\hline
\end{tabular}

Org. dos autores

\section{RESULTADOS E DISCUSSÕES}

As séries temporais do MODIS EVI2 representativas de pastagem cultivada (Ap), cultura agrícola (Ac), florestal estacional semidecidual aluvial (Fa) e savana gramíneo-lenhosa (Sg) são mostradas na Figura 2. Todas as quatro classes apresentaram efeitos marcantes da sazonalidade climática, notadamente para Ac. De uma forma geral, independentemente do dia do ano, os valores de EVI2 do Ap e do Sg foram menores que os do $\mathrm{Fa}$, por causa dos seus índices mais baixos de biomassa. Os valores de EVI2 da cultura agrícola também se destacam dos demais por apresentarem os índices mais altos no período chuvoso e os mais baixos no período seco. No entanto, mesmo na estação seca, supõe-se que havia algum tipo de cobertura vegetal verde nessa área de cultura agrícola no período analisado, uma vez que os valores de EVI são todos diferentes de zero (menor valor: 23 de setembro de 2001, EVI2 = 0,0129). O caráter bimodal das curvas da cultura agrícola sugere que a área apresenta dois plantios por ano (exemplo, milho safrinha) ou há plantio de cobertura verde logo após a colheita (exemplo, soja seguido de milheto).

Uma técnica de potencializar a discriminação de alvos é acumular os valores da série temporal do EVI2 (Figura 3). Assim, o EVI2 de 16 de fevereiro de 2000 de Ap apresentou variação de EVI2 inferior a 0,07 em relação aos demais alvos: 0,$063 ; 0,059$; e 0,063 de diferença em relação a $\mathrm{Ac}, \mathrm{Fa}$ e $\mathrm{Sg}$, respectivamente. Após acumular os valores de EVI2 para toda a série analisada (até 26 de dezembro de 2010), as diferenças aumentaram para 8,$26 ; 25,45$; e 17,52, respectivamente. Esses valores acumulados permitem que cada classe dificilmente seja confundida com outras classes, conforme ressaltado por Rosendo e Rosa (2005). Na realidade, essa estratégia de acumular dados espectrais para melhorar a capacidade de discriminação de alvos foi inicialmente proposto por Ratana et al. (2005) e o seu desempenho tem sido confirmado por outros autores como Rosendo e Rosa (2005) e Hermuche e Sano (2011). No entanto, cabe realçar que se acumulando os valores da série temporal somente dentro de um ciclo hidrológico pode-se obter melhores resultados.

Figura 2: Séries temporais do MODIS EVI2 de pastagem cultivada (Ap), cultura agrícola (Ac), floresta estacional semidecidual aluvial $(\mathrm{Fa})$ e savana gramíneo-lenhosa $(\mathrm{Sg})$ da área de estudo.

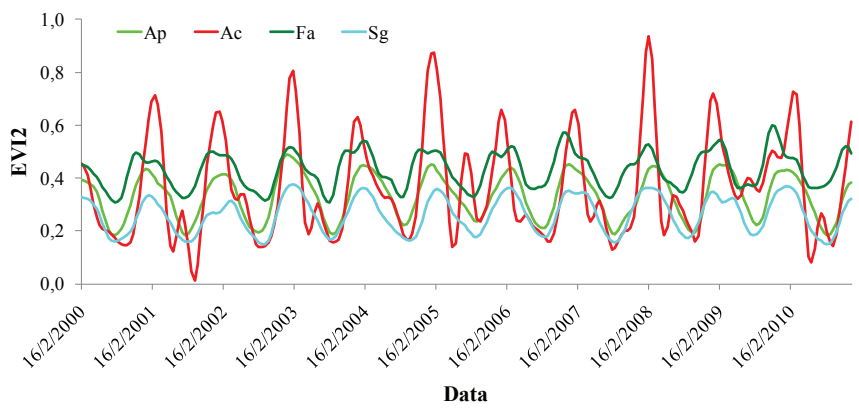

Org. dos autores 
Figura 3: EVI2 acumulado de pastagem cultivada (Ap), cultura agrícola (Ac), floresta estacional semidecidual aluvial (Fa) e savana gramíneo-lenhosa (Sg) da área de estudo para o período de 2000 a 2010.

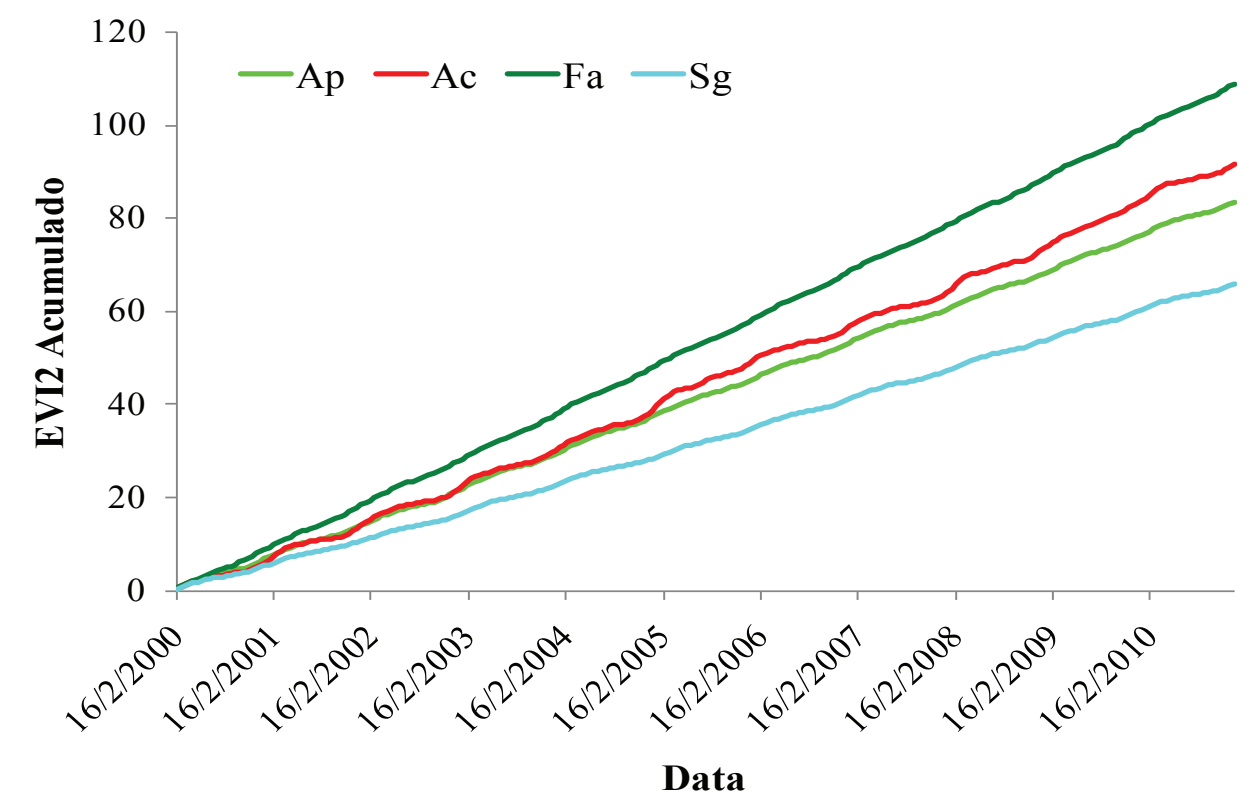

Org. dos autores.

A localização dos 25 pontos de pastagens cultivadas selecionadas para esse estudo nos três municípios em questão é mostrada na Figura 4. Um ponto esteve localizado no município de Araguari, oito pontos no município de Uberaba e 16 pontos no município de Uberlândia. Todos os pontos corresponderam à pastagem do tipo Brachiaria.

Figura 4. Mapa de localização dos pontos de amostragem nos municípios de Araguari, Uberaba e Uberlândia.

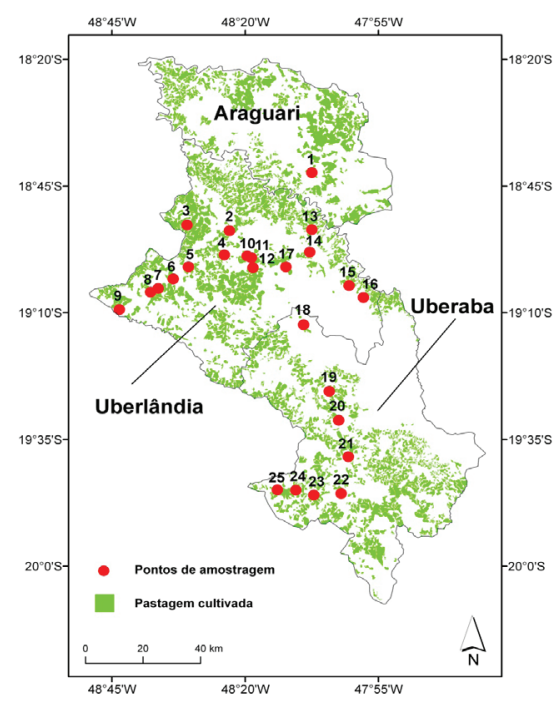

Org. dos autores. 
Resultados da análise de agrupamento hierárquico na forma de dendrograma (Figura 5) indicam que a maioria das 25 pastagens selecionadas apresentaram baixa similaridade entre si em termos de assinatura espectro-temporal. As exceções são as pastagens 1 e 9; 23, 24 e 25; e 20 e 22 que apresentaram distância euclidiana relativa inferior a 0,5 . Pela Figura 6 , pode-se confirmar a similaridade espectral dessas duas pastagens ao longo de toda a série temporal. Esse resultado indica que a maioria das pastagens cultivadas da área de estudo apresentou particularidades ao longo dos 10 anos considerados. Essa particularidade pode estar relacionada com diferenças nos manejo das pastagens tais como diferenças no número de animais/hectares, práticas de calagem e remoção de plantas invasoras. Diferenças nas datas de abertura de pastagens e de renovação das pastagens também podem ter contribuído para o baixo agrupamento de classes mostrado no dendrograma. Novamente cabe destacar que se for utilizado dados originados dentro de apenas um ciclo hidrológico talvez fosse possível conseguir maior similaridade.

Figura 5. Dendrograma obtido da análise de agrupamento hierárquico utilizando, como variáveis, as séries temporais do MODIS

EVI2 do período de 2000 a 2010.

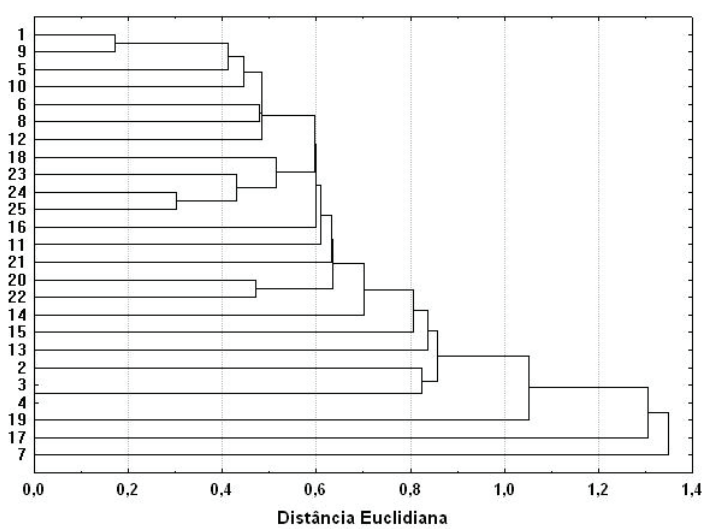

Org. dos autores.

Informações adicionais que podem ser extraídas das séries temporais relativas às pastagens cultivadas do Triângulo Mineiro são mostradas nas próximas quatro figuras. Nessas figuras, a série temporal da pastagem 25 (mostrada na Figura 2) é comparada com quatro outras séries temporais das pastagens 13, 14, 17 e 22 . Na Figura 7, percebe-se que a pastagem cultivada foi implementada a partir de 2004, provavelmente substituindo uma área natural, ocupada por uma floresta estacional (valores máximos de EVI2 em torno de 0,55).

Figura 6. Séries temporais do MODIS EVI2 das pastagens 1 e 9, ilustrando a similaridade espectral elevada entre elas.

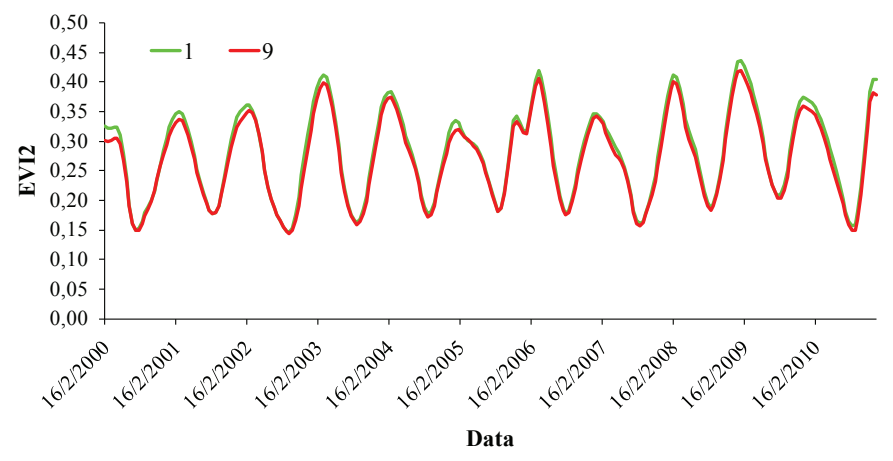

Org. dos autores. 
Figura 7. Séries temporais do MODIS EVI2 das pastagens 13 e 25, ilustrando possível implementação de pastagem cultivada em 2004, substituindo uma área de Cerrado natural.

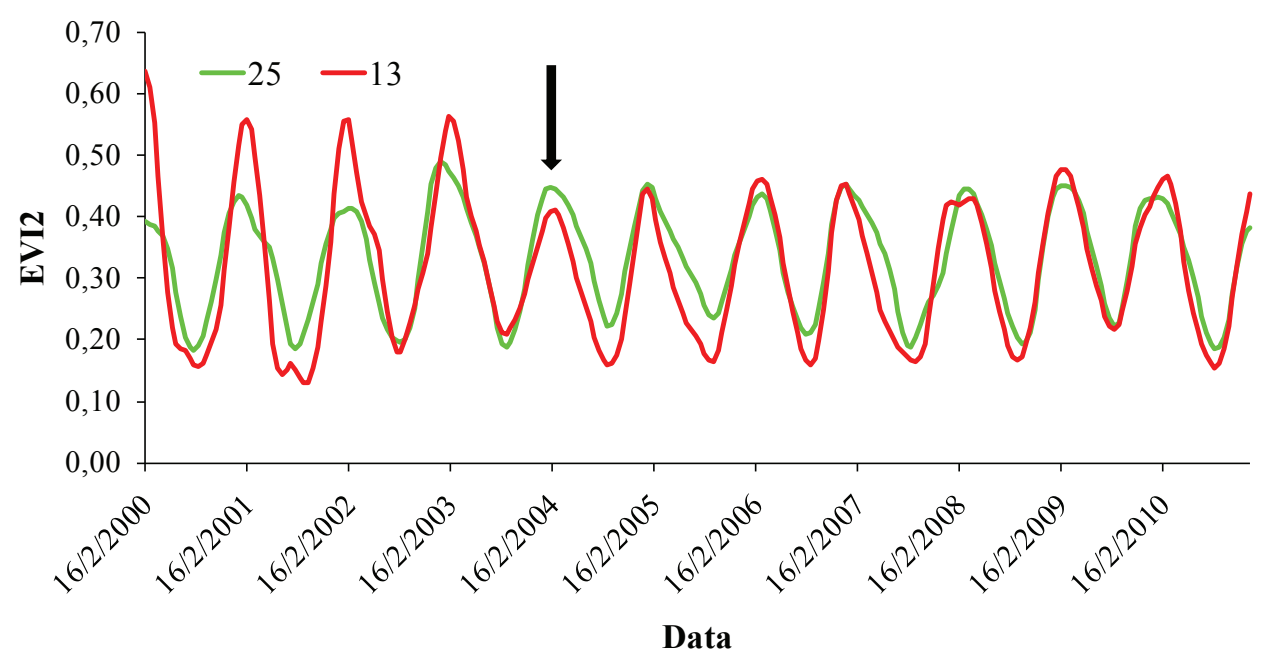

Org. dos autores.

A pastagem 14 diferencia-se da pastagem 25 por apresentar, sistematicamente até o final de 2006, valores mais baixos de EVI2 (decréscimo da ordem de 0,05 a 1,0) (Figura 8). A partir de 2007, essa diferença não é mais acentuada, sendo inclusive maior em algumas datas. Já a área coberta pela pastagem 17 (Figura 9) mostra que houve substituição por cultura agrícola (valores máximos de EVI2 superiores a 0,70) no período de 2004 a 2006. Finalmente, na Figura 10, é destacada uma situação em que os valores de EVI2 da pastagem 22 são sistematicamente mais altos no período seco.

Figura 8. Séries temporais do MODIS EVI2 das pastagens 14 e 25, ilustrando valores de EVI2 sistematicamente mais baixos até o início de 2007 para a pastagem 14.

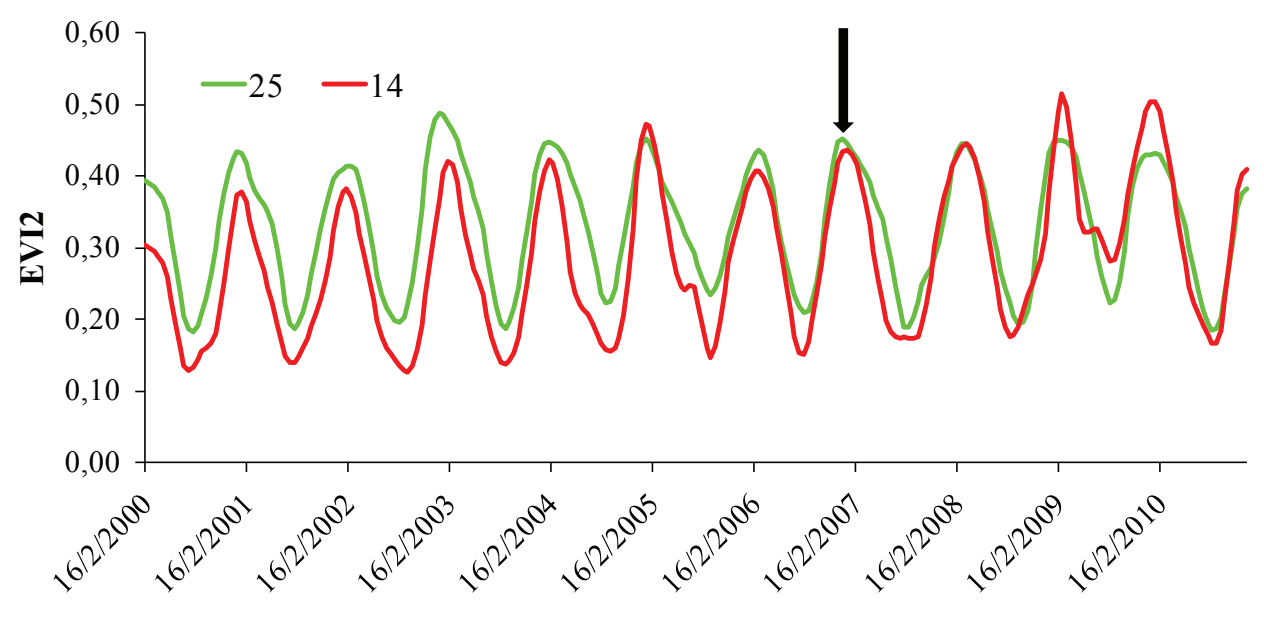

Data

Org. dos autores. 
Figura 9. Séries temporais do MODIS EVI2 das pastagens 17 e 25, ilustrando a substituição da pastagem por cultura agrícola no período de 2004 a 2007 na pastagem 17.

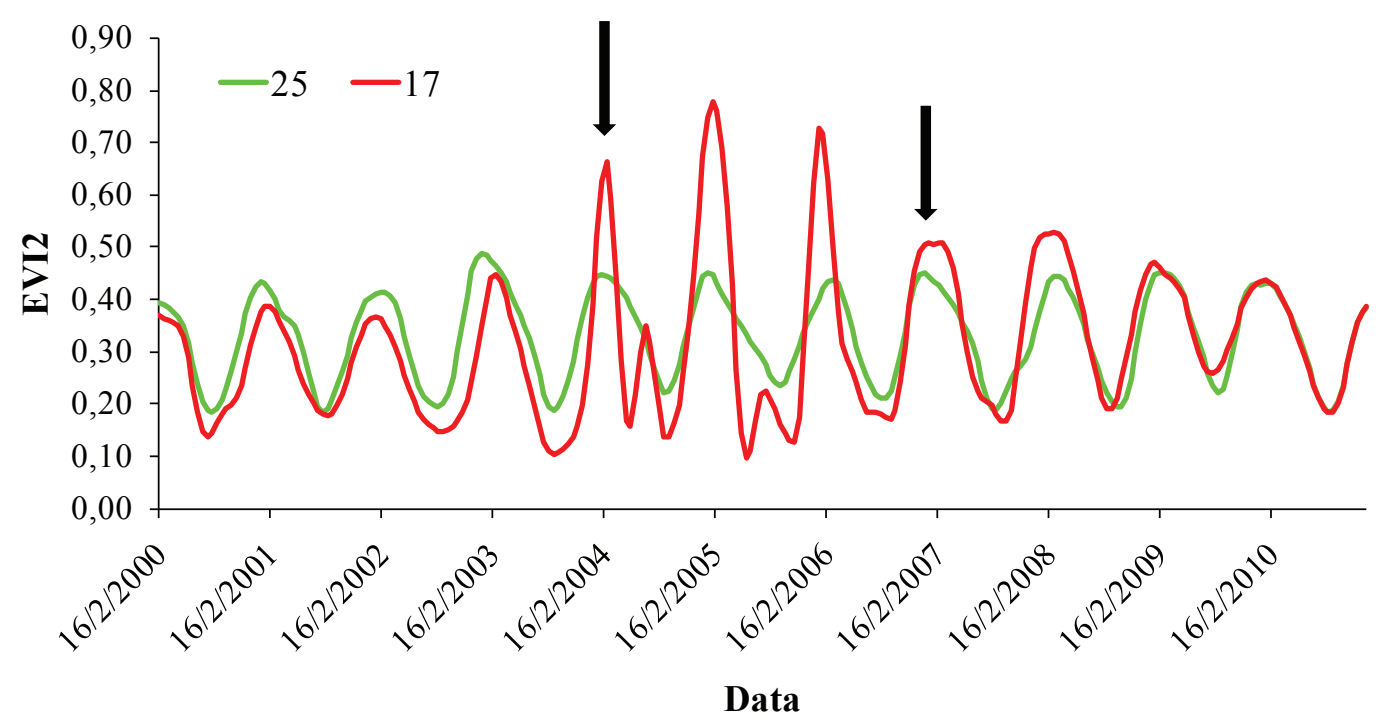

Org. dos autores.

Figura 10. Séries temporais do MODIS EVI2 das pastagens 22 e 25, destacando uma situação em que os valores de EV2 são sistematicamente mais elevados no período seco.

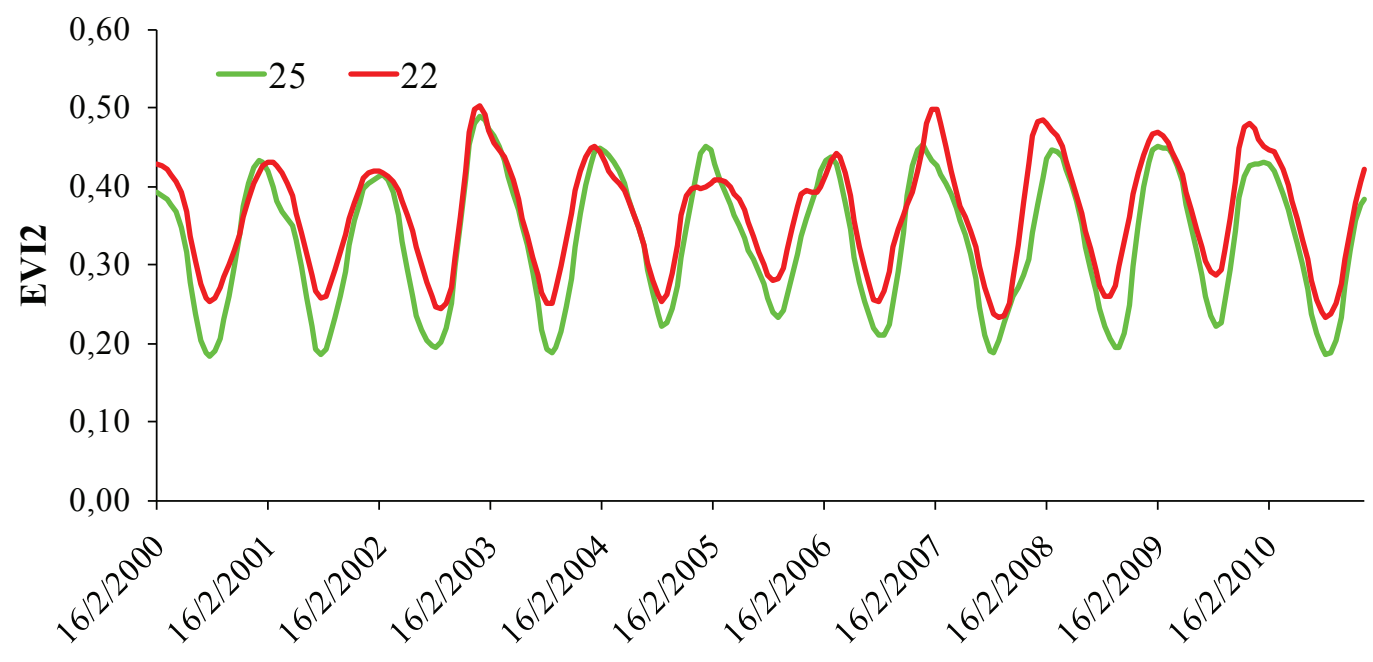

Data

Org. dos autores. 


\section{CONCLUSÕES}

A análise da série temporal do MODIS EVI2 indicou que:

a) é possível discriminar pastagens cultivadas de outras classes de cobertura de terras da região;

b) a somatória de valores de EVI2 de toda a série histórica pode aumentar consideravelmente a capacidade de discriminação de pastagens cultivadas;

c) a maioria das pastagens cultivadas apresentaram assinaturas espectro-temporais distintas; e

d) a substituição da pastagem por outros tipos de cobertura de terras (por exemplo, culturas agrícolas e vice-versa) é facilmente identificada na série temporal do MODIS EVI2.

\section{AGRADECIMENTOS}

Esse estudo fez parte da dissertação de mestrado da primeira autora no programa de pós-graduação em Geociências Aplicadas da Universidade de Brasília, Brasília/DF. Os autores agradecem à equipe do Laboratório de Agricultura e Floresta do INPE, em especial, ao Bernardo Rudorff, Egídio Arai e Ramon Freitas, pelo auxílio na disponibilização da série temporal do MODIS EVI2.

\section{REFERÊNCIAS}

BRITO, J.L.S.; PRUDENTE, T.D. Mapeamento do uso da terra e cobertura vegetal do município de Uberlândia - MG, utilizando imagens CCD/CBERS 2. Caminhos da Geografia, v. 13, n. 15, p. 144-153, 2005a.

BRITO, J.L.S.; PRUDENTE, T.D. Análise temporal do uso do solo e cobertura vegetal do município de Uberlândia-MG, utilizando imagens ETM+/Landsat 7. Sociedade \& Natureza, v. 17, n. 32, p. 37-46, 2005 b.
FREITAS, R.M.; ARAI, E.; ADAMI, M.; FERREIRA, A.S.; SATO, F.Y.; SHIMABUKURO, Y.E.; ROSA, R.R.; ANDERSON, L.O.; RUDORFF, B.F.T. Virtual laboratory of remote sensing time series: visualizations of MODIS EVI2 data set over South America. Journal of Computacional Interdisciplinary Sciences, v. 2 , n. 1, p. 57-68, 2011.

HERMUCHE, P.M.; SANO, E.E. Identificação da floresta estacional decidual no Vão do Paranã, estado de Goiás, a partir da análise da reflectância acumulada de imagens do sensor ETM+/Landsat-7. Revista Brasileira de Cartografia, n. 63, n. 3, p. 415-425.

HUETE, A.; DIDAN, K.; MIURA, T.; RODRIGUEZ, E.P.; GAO, X.; FERREIRA, L.G. Overivew of the radiometric and biophysical performance of the MODIS vegetation indices. Remote Sensing of Environment, v. 83, p. 195-213, 2002.

JIANG, Z.; HUETE, A.R.; DIDAN, K.; MIURA, T. Development of a two-band enhanced vegetation index without a blue band. Remote Sensing of Environment, v. 112, n. 10, p. 3833-3845, 2008.

JUSTICE, C.O.; TOWNSHEND, J.R.G.; VERMOTE, E.F.; MASUOKA, E.; WOLFE, R.E.; SALEOUS, N.; ROY, D.P.; MORISETTE, J.T. An overview of MODIS land data processing and product status. Remote Sensing of Environment, v. 83, n. 1-2, p. 3-15, 2002.

INPE. Instituto Nacional de Pesquisas Espaciais. $\mathrm{Ca}$ tálogo de imagens Landsat. Disponível em: $<\mathrm{http}: / /$ www.dgi.inpe.br>. Acesso em: 20 mar. 2011.

MELO, E.O; FEHR, M. O uso atual do solo e da água na bacia do ribeirão Piçarrão-Araguari-MG-Brasil. Investigaciones Geograficas, v. 72, p. 39-48, 2010.

MIURA, T.; YOSHIOKA, H.; FUJIWARA, K.; YAMAMOTO, H. Inter-comparison of ASTER and MODIS surface reflectance and vegetation index products for synergistic applications to natural resource monitoring. Sensors, v. 8, p. 2480-2499, 2008. 
MMA. Ministério do Meio Ambiente. Mapa de cobertura vegetal dos biomas brasileiros. Brasília: MMA/Secretaria de Biodiversidade e Florestas. Disponível em: <http://www.mma.gov.br/sitio/index. php?ido $=$ conteudo.monta\&idEstrutura $=72 \&$ idMe nu $=3813>$. Acesso em: 28 jan. 2012.

MOITANETO, J.M.; MOITA, G.C. Uma introdução à análise exploratória de dados multivariados. Química Nova, v. 21, n. 4, p. 467-469, 1998.

RATANA, P.; HUETE, A.R.; FERREIRA, L.G. Analysis of Cerrado physiognomies and conversion in the MODIS seasonal-temporal domain. Earth Interactions, v. 9, Paper no. 3, 22 p., 2005.

RIBEIRO, J.F.; WALTER, B.M.T. Fitofisionomias do Bioma Cerrado. In: SANO, S.M.; ALMEIDA, S.P.; RIBEIRO, J.F. (eds.) Cerrado. Ambiente e Flora. Planaltina: Embrapa Cerrados, p. 89-166, 2008.

ROSENDO, J.S.; ROSA, R. A utilização de sensores com resolução moderada (MODIS) no estudo da vegetação na bacia do rio Araguari - MG. Sociedade \& Natureza, v. 17, n. 33, p. 91-104, 2005.

SANO, E.E.; ROSA, R.; BRITO, J.L.S.; FERREIRA, L.G. Land cover mapping of the tropical savanna region in Brazil. Environmental Monitoring and Assessment, v. 166, p. 113-124, 2010. 